Supplement of Nat. Hazards Earth Syst. Sci., 20, 3197-3214, 2020

https://doi.org/10.5194/nhess-20-3197-2020-supplement

(C) Author(s) 2020. This work is distributed under

the Creative Commons Attribution 4.0 License.

(c) (1)

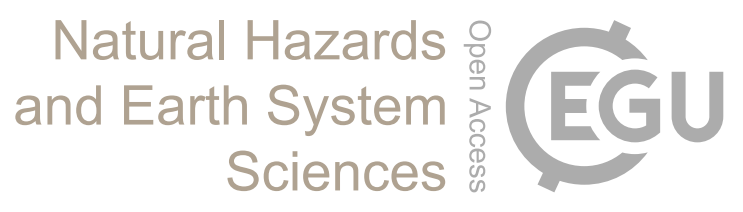

Supplement of

\title{
A systematic exploration of satellite radar coherence methods for rapid landslide detection
}

Katy Burrows et al.

Correspondence to: Katy Burrows (katy.a.burrows@durham.ac.uk)

The copyright of individual parts of the supplement might differ from the CC BY 4.0 License. 


\begin{tabular}{|c|c|c|c|c|c|c|c|c|c|c|c|c|c|}
\hline & \multirow{3}{*}{$\begin{array}{r}\text { Event } \\
\text { Satellite } \\
\text { Track number }\end{array}$} & \multicolumn{4}{|c|}{ Hokkaido } & \multicolumn{3}{|c|}{ Nepal } & \multicolumn{3}{|c|}{ Lombok } & \multirow{2}{*}{\multicolumn{2}{|c|}{$\frac{\text { Lombok } 2}{\mathrm{~S}-1}$}} \\
\hline & & \multicolumn{2}{|c|}{ S-1 } & \multicolumn{2}{|c|}{$A-2$} & \multicolumn{2}{|c|}{ S-1 } & $A-2$ & \multicolumn{2}{|c|}{ S-1 } & \multirow{2}{*}{$\frac{A-2}{129 a}$} & & \\
\hline & & 068a & 046d & $116 a$ & $018 \mathrm{~d}$ & 085a & 019d & $157 a$ & $156 a$ & $032 d$ & & $156 a$ & $032 d$ \\
\hline \multirow{3}{*}{ 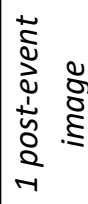 } & ARIA & 0.103 & 0.099 & 0.179 & 0.296 & 0.005 & 0.002 & 0.045 & 0.002 & 0.001 & 0.009 & 0.002 & 0.004 \\
\hline & Bx-S & 0.120 & 0.116 & 0.103 & 0.084 & 0.008 & 0.009 & - & 0.002 & 0.001 & 0.009 & 0.010 & 0.004 \\
\hline & Waiting time (days) & 8 & 0 & 1 & 1 & 8 & 4 & 7 & 3 & 0 & 13 & 1 & 4 \\
\hline \multirow{4}{*}{ 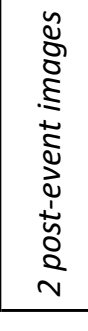 } & $\mathrm{PECl}$ & 0.271 & 0.273 & 0.143 & 0.195 & 0.009 & 0.005 & 0.022 & 0.015 & 0.003 & 0.018 & 0.005 & 0.005 \\
\hline & $\Delta C$ C_sum & 0.180 & 0.191 & 0.179 & 0.257 & 0.006 & 0.002 & $0.057 \mid$ & 0.003 & 0.002 & 0.016 & 0.004 & 0.005 \\
\hline & $\Delta C_{-}$max & 0.206 & 0.211 & 0.143 & 0.295 & 0.007 & 0.002 & 0.051 & 0.014 & 0.002 & 0.014 & 0.004 & 0.005 \\
\hline & Waiting time (days) & 20 & 12 & 15 & 15 & 20 & 16 & 91 & 9 & 6 & 153 & 7 & 10 \\
\hline & Landslide fraction & 0.106 & 0.116 & 0.111 & 0.104 & 0.004 & 0.001 & 0.006 & 0.001 & 0.001 & 0.002 & 0.002 & 0.003 \\
\hline
\end{tabular}

Precision-recall values equivalent to the ROC AUC values given in Figure $2 a$ of the main text. In each column, the colour scale runs from red (the landslide fraction) up to green ( $3 x$ the landslide fraction) The landslide fraction represents the fraction of pixels in the validation dataset containing more than $25 \%$ landslide by area 
Hokkaido Nepal Lombok-1 Lombok-2

1\% S068a S046d A116a A018d S085a S019d A157a S156a S032d A129a S156a S032d

\begin{tabular}{|c|c|c|c|c|c|c|c|c|c|c|c|c|}
\hline ARIA & 50 & 0.54 & 0.65 & 0.87 & 0.59 & 0.70 & 0.79 & 0.58 & 0.47 & 0.74 & 0.57 & 0.39 \\
\hline$B x-S$ & .57 & 0.5 & 0.45 & & & & - & & & 0.66 & .69 & 0.61 \\
\hline $\mathrm{Cl}$ & & & & & & & & & & & & 58 \\
\hline & & & 0.66 & 0 & & & & & & 0.75 & 0.63 & 0.47 \\
\hline $\mathrm{cm}$ & 0.64 & 0.64 & 0.60 & 0.85 & 0.61 & 0.71 & 0.81 & 0.60 & 0.48 & 0.76 & 0.65 & 0.49 \\
\hline
\end{tabular}

\begin{tabular}{|c|c|c|c|c|c|c|c|c|c|c|c|c|}
\hline $10 \%$ & & & & & & & & & & & & \\
\hline ARIA & 0.51 & 0.55 & 0.67 & 0.88 & 0.59 & 0.69 & 0.80 & 0.62 & 0.50 & 0.82 & 0.52 & 0.41 \\
\hline$B x-S$ & 0.58 & 0.57 & 0.49 & 0.43 & 0.64 & 0.72 & - & 0.61 & 0.57 & 0.76 & 0.71 & 0.63 \\
\hline $\mathrm{PECl}$ & 0.72 & 0.71 & 0.65 & 0.77 & 0.65 & 0.67 & 0.79 & 0.55 & 0.58 & 0.87 & 0.64 & 0.60 \\
\hline DC_sum & 0.67 & 0.67 & 0.68 & 0.86 & 0.65 & 0.71 & 0.86 & 0.64 & 0.53 & 0.86 & 0.61 & 0.49 \\
\hline DC_max & 0.68 & 0.69 & 0.62 & 0.87 & 0.61 & 0.70 & 0.84 & 0.65 & 0.52 & 0.86 & 0.63 & 0.51 \\
\hline
\end{tabular}

\begin{tabular}{|c|c|c|c|c|c|c|c|c|c|c|c|c|}
\hline $25 \%$ & & & & & & & & & & & & \\
\hline ARIA & 0.52 & 0.57 & 0.70 & 0.89 & 0.59 & 0.69 & 0.81 & 0.64 & 0.56 & 0.88 & 0.49 & 0.45 \\
\hline$B x-S$ & 0.59 & 0.58 & 0.52 & 0.44 & 0.66 & 0.74 & - & 0.64 & 0.55 & 0.84 & 0.67 & 0.60 \\
\hline $\mathrm{PECl}$ & 0.79 & 0.78 & 0.66 & 0.80 & 0.66 & 0.68 & 0.81 & 0.64 & 0.69 & 0.93 & 0.68 & 0.68 \\
\hline DC_sum & 0.73 & 0.73 & 0.70 & 0.88 & 0.66 & 0.71 & 0.88 & 0.71 & 0.62 & 0.92 & 0.63 & 0.57 \\
\hline DC_max & 0.74 & 0.75 & 0.64 & 0.89 & 0.62 & 0.71 & 0.85 & 0.72 & 0.62 & 0.92 & 0.65 & 0.59 \\
\hline
\end{tabular}

\begin{tabular}{|c|c|c|c|c|c|c|c|c|c|c|c|c|}
\hline $50 \%$ & & & & & & & & & & & & \\
\hline ARIA & 0.57 & 0.64 & 0.79 & 0.91 & 0.59 & 0.69 & 0.84 & - & - & - & - & - \\
\hline$B x-S$ & 0.63 & 0.63 & 0.64 & 0.49 & 0.70 & 0.80 & - & - & - & - & - & - \\
\hline $\mathrm{PECl}$ & 0.98 & 0.96 & 0.68 & 0.88 & 0.67 & 0.69 & 0.88 & - & - & - & - & - \\
\hline DC_sum & 0.91 & 0.90 & 0.77 & 0.93 & 0.68 & 0.73 & 0.94 & - & - & - & - & - \\
\hline DC_max & 0.92 & 0.92 & 0.71 & 0.95 & 0.63 & 0.73 & 0.90 & - & - & - & - & - \\
\hline
\end{tabular}

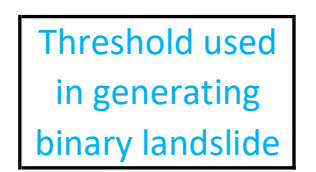

\begin{tabular}{|r|cccc|}
\hline \multicolumn{5}{|c|}{$\begin{array}{c}\text { Fraction of total landslide area included in } \\
\text { analysis }\end{array}$} \\
& Hokkaido & Nepal & Lombok-1 & Lombok-2 \\
\cline { 1 - 1 } $1 \%$ & 100 & 99 & 96 & 97 \\
\cline { 1 - 1 } $10 \%$ & 94 & 74 & 45 & 47 \\
\cline { 1 - 1 } $25 \%$ & 73 & 39 & 14 & 15 \\
\cline { 1 - 1 } $50 \%$ & 30 & 15 & 2 & 3 \\
\hline
\end{tabular}

